\title{
Scherrer formula: estimation of error in determining small nanoparticle size
}

\author{
A. S. Vorokh \\ Institute of Solid State Chemistry of the Ural Branch of the Russian Academy of Sciences, \\ 91 Pervomaiskaya st., Ekaterinburg, Russia \\ vorokh@ihim.uran.ru
}

PACS 61.05.cc, 61.46.Df

DOI 10.17586/2220-8054-2018-9-3-364-369

The lower limit of the applicability of the Scherrer formula has been established by calculating the diffraction patterns from model nanoparticles by the Debye formula. Particle size was calculated using the Scherrer formula for different $h k l$-peaks. The obtained data of particle sizes were compared with "real" sizes of model particles in the same $h k l$-directions. The form-factor $K_{h k l}$ was analyzed as main correction of Scherrer formula. It was shown that the Scherrer formula error increases nonlinearly at particle sizes less than $4 \mathrm{~nm}$. For any $h k l$ direction, the absolute error of average particle size determination using formula does not exceed $0.3 \mathrm{~nm}$. Analysis shows that average particle size can be determined by Scherrer formula from single diffraction peak of experimental pattern for center-symmetrical particles.

Keywords: Scherrer formula, nanoparticle size, Scherrer limit, Debye equation.

Received: 30 November 2017

Revised: 7 February 2018

Final revision: 3 April 2018

\section{Introduction}

One of the most important physical properties of nanostructured materials is particle size. Among the techniques developed for nano-scale particle size determination, the analysis of average size of coherent scattering regions from X-ray powder diffraction reflection broadening by the Scherrer formula is the simplest and most convenient [1].

Although there are numerous refinements of the formula, the question of the limits of its applicability is still unclear. The theoretical upper limit of applicability of the Scherrer formula was analyzed in detail and was found up to $600 \mathrm{~nm}$ for $\mathrm{LaB}_{6}, \mathrm{Si}$ and $\mathrm{CeO}_{2}$ [2] and $11.9 \%$ from extinction length in general case [3]. Comparison of different experimental methods based on the Scherrer formula with TEM data for different materials showed that crystallite size values are the closest when particle sizes are less than $60 \mathrm{~nm}$ [4]. The Scherrer formula has no the lower limit of applicability but the question remains: what is the error of the formula when particle size decreases to zero $\mathrm{nm}$ ? In the work [5] experimental investigation of $\mathrm{TiO}_{2}$ anatase nanoparticles shows that absolute divergence is $0.36 \mathrm{~nm}$ for the crystallites smaller than $10 \mathrm{~nm}$ and $2.4 \mathrm{~nm}$ for the the crystallites larger than $15 \mathrm{~nm}$.

In this work we proposed a facile theoretical analysis of the application of the Scherrer formula to diffraction reflections calculated by the Debye equation from model particles, and the contribution of the form-factor while using the Scherrer formula is evaluated. The calculation by the Debye formula is a direct result of scattering on an ensemble of atoms (irrespective of the character of ordering), which allows evaluation of the Scherer formula accuracy. Moreover, the Debye formula was deduced in the framework of the same two assumptions that were used to derive the Scherrer formula [6]. Independence from translational symmetry allows one to use the Debye equation for investigating the non-crystalline materials [7], stacking faults [8,9], surface relaxation [10],poorly crystalline materials [11]. Currently, the powerful software programs as DIANNA [12,13], DEBUSSY [14] allows to apply Debye function analysis (DFA) as routine procedure for structure interpretation [15]. Nevertheless, the most material scientists uses the Scherrer formula as the simplest method of particle size determination.

The formula proposed by P. Scherrer in 1918 [16] describes the broadening of diffraction reflection peaks $\beta$ as a function of the average particle size $D$ :

$$
\beta=K \frac{\lambda}{D \cos \theta},
$$

where $\lambda$ is the $\mathrm{X}$-ray wave length, $\theta$ is the Bragg reflection angle, and $K=2 \sqrt{(\ln 2) / \pi}$ is a constant. Formula (1) was deduced with two assumptions:

1) an X-ray wave is singly scattered on atoms of a substance (so-called kinematical approximation), and dynamic effects exert a negligibly small influence on the form of the diffraction pattern; 
2) a material is an "ideal powder" consisting of an infinite number of identical particles located chaotically in space, which allows the powder crystallite size and shape and sample texture distribution to be neglected. The particles were supposed by P. Scherrer [16] to have a cubic shape and cubic structure.

Taking into account the above mentioned assumptions, formula (1) should presume no rigorous experimental check, and its application for the processing of experimental diffraction data has mainly an evaluative character. The error of formula (1) can be established only by comprehensive consideration of all peculiarities of the equation and by application to diffraction patterns calculated from model powder.

Let us consider the formula itself in detail. As given in [16], the formula included two ambiguous values. Firstly, the parameter $\beta$, which the author [16] designated as halfwidth in "the generally accepted sense" without specifying whether this was full width at half maximum (FWHM) or integral breadth (IB) equal to the area under the peak related to its maximal value. Secondly, the value of constant $K$ called subsequently as the Scherrer constant or form-factor was not clear from the article. The work [16] interpreted the constant $K$ as a relating factor between the FWHM and IB of the Gaussian function: $\mathrm{FWHM}_{\mathrm{G}}=2 \sqrt{\frac{\ln 2}{\pi}} \mathrm{IB}_{\mathrm{G}}=K \cdot \mathrm{IB}_{\mathrm{G}}$, suggesting that Scherrer supposed the diffraction peak to be approximated by the Gaussian, consequently, $\beta=$ FWHMG. Then, the Scherrer formula may be written as $\mathrm{FWHM}_{\mathrm{G}}=K \frac{\lambda}{D \cos \theta}$, where $K=2 \sqrt{\frac{\ln 2}{\pi}}$. The analogous notation $\mathrm{IB}_{\mathrm{G}}=\frac{\lambda}{D \cos \theta}$ reflects the physical meaning of the formula more clearly - the area under the diffraction curve is related with the radial distribution of atoms in the particle and, accordingly, with the size of this particle.

The factor $K=\sqrt{3 / \pi} \approx 0.977$ as a necessary numerical correction appeared for the first time in work [17], where N. Selyakov presented the first detailed derivation of formula (1) for particles of trigonal structure. In the subsequent works devoted to this subject $[18,19]$, the values of the form-factor $K$ were proposed for particles of different shape depending on $h k l$ directions. According to [19], for a powder consisting of equal cubic crystallites, the coefficient $K$ for different crystallographic indices $(h k l)$ of the cubic crystal lattice was calculated by the formula:

$$
K_{h k l}=\frac{6|h|^{3}}{\left(h^{2}+k^{2}+l^{2}\right)^{1 / 2}\left(6 h^{2}-2|h k|+|k l|-2|h l|\right)} .
$$

In this study, cubic particles of simple cubic structure with the space group $\operatorname{Pr} \overline{3} m$ have been chosen as model objects, since the Scherrer formula was derived exactly for such particles. We compared the particle size values calculated by equation (1) both without using the form-factor $K_{h k l}$ and with the correction expressed by equation (2). For this purpose, we calculated the diffraction patterns from model particles by the Debye formula [20]:

$$
I(q)=\sum_{j=1}^{N} \sum_{k=1}^{N} f_{j}(q) f_{k}(q) \frac{\sin \left(q R_{j k}\right)}{q R_{j k}},
$$

where $I(q)$ is the scattering intensity, $R_{j k}$ is the distance between the $j$-th and the $k$-th atoms; $q$ is the modulus of the scattering vector; and $f_{j}(q)$ is the atomic scattering vector for the $j$-th atom. The scattering intensity on "ideal powder" is composed of scattering intensities on each individual powder particle and, consequently, is determined by integration over all possible positions of this particle-object in space.

The model cubic particles were set by generating the atomic coordinates with the use of a simple cubic lattice with a distance between nearest atoms equal to $0.3 \mathrm{~nm}$. The atomic scattering factors $f_{j}(2 \theta)=f_{j}(q)$ of model particles were calculated by approximating the tabular data for silicon atoms [21]. In the calculation of the scattering intensity measured in a real X-ray diffraction experiment by the Bragg-Brentano method, angular scattering factors should be also taken into account along whith the structural factor: the geometric factor $G$, the Lorentz factor $L$ and the polarization factor $P(\theta)=\left(1+\cos ^{2} 2 \theta\right) / 2$. The correction is made by multiplying the intensity by the angular factor, $L P G(\theta)=\frac{1+\cos ^{2} 2 \theta}{4 \cdot \sin 2 \theta \cdot \sin \theta}$. The diffraction patterns were calculated for $\mathrm{Cu} K \alpha$ radiation with wavelength $0.154 \mathrm{~nm}$.

Diffraction peak profiles can be approximate by different methods with the highest degree of accuracy [22-24]. For accurate description of the peak shape, each diffraction peak was approximated by the pseudo-Voigt function by the formula:

$$
V(\theta)=c a\left[1+\frac{\left(\theta-\theta_{0}\right)^{2}}{\theta_{\mathrm{L}}^{2}}\right]^{-1}+(1-c) a \exp \left[-\frac{\left(\theta-\theta_{0}\right)^{2}}{2 \theta_{\mathrm{G}}^{2}}\right]=c \cdot l(\theta)+(1-c) \cdot g(\theta),
$$


where $l(\theta)$ is the Lorentz function; $g(\theta)$ is the Gaussian function; $c$ is the relative contribution of the Lorentz function to the total reflection intensity; $\theta_{\mathrm{L}}$ and $\theta_{\mathrm{G}}$ are the Lorentz and Gaussian distribution parameters, respectively; $a$ is the normalizing factor of intensity; and $\theta_{0}$ is the position of the pseudo-Voigt function maximum. The exact $\mathrm{FWHM}_{V}$ value is determined by the solution of the nonlinear equation

$$
V\left(\theta_{h}\right)=\frac{a c}{1+\left(\theta_{h} / b\right)^{2}}+a(1-c) \exp \left[-\frac{\left(\theta_{h} / b\right)^{2}}{2}\right]=\frac{a}{2},
$$

which is not solved analytically, but the solution of this equation is satisfactorily described by the quadratic dependence $\theta_{h}=2.355-0.276 c-0.079 c^{2}$, consequently, $\mathrm{FWHM}_{\mathrm{V}}=b\left(2.355-0.276 c-0.079 c^{2}\right)$. The error of such approximation is about $0.01 \%$. The numerical values of the parameters $a, b=\theta_{\mathrm{L}}=\theta_{\mathrm{G}}, c, 2 \theta_{0}$ of each reflection were obtained in the framework of the application program SigmaPlot.

Figure 1 displays the results of the calculations for the X-ray diffraction patterns. The diffraction patterns were calculated for cubic nanoparticles containing 8, 27, 64, 125, 256, 1728 or 17576 atoms. The number of unit cell translations was $1,2,3,4,5,11$ and 25 , respectively. With an increase in the nanoparticle size, the broad reflections typical for amorphous substance tend to turn into narrow peaks peculiar to coarse-crystalline material.

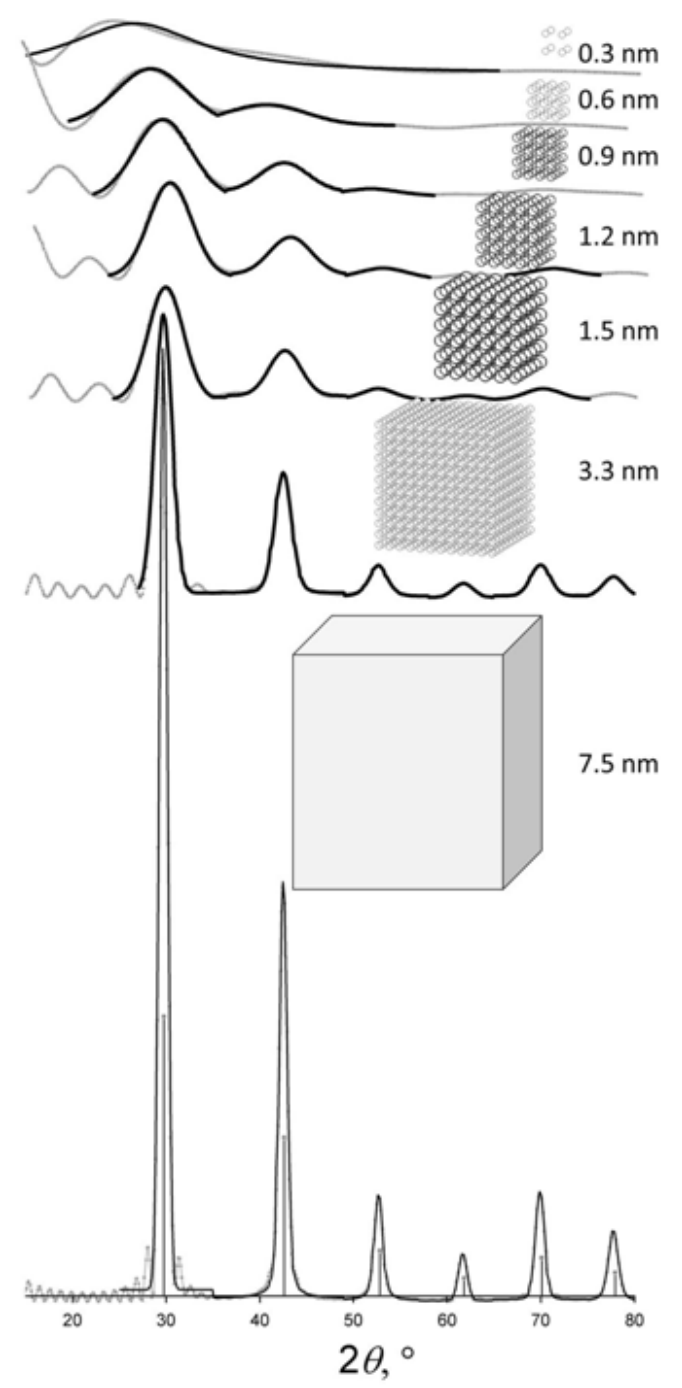

FIG. 1. Diffraction curves calculated by the Debye formula (grey) for cubic particles of the cubic unit cell structure, and approximation of reflections by the pseudo-Voigt function (black). The corresponding model particles are shown next to the diffraction patterns, the edge length is given in $\mathrm{nm}$ 
The particle size was calculated by formula (1) $D^{S c h}=\lambda / \mathrm{FWHM}_{\mathrm{V}} \cos \theta_{0}$ and also with the correction for $K_{h k l}: D_{h k l}^{S c h}=K_{h k l} \lambda / \mathrm{FWHM}_{\mathrm{V}} \cos \theta_{0}$. The coefficient $K_{a}$ for the determination of the linear size $D^{D e b}=K_{a} \cdot a$ of a cubic particle with edge side $a$ in a given direction $(h k l)$ was calculated in a similar way. For example, the linear particle size in the direction (111) is equal to the body diagonal of a cube $a \sqrt{3}$. This makes it possible to relate the sizes of a preset model cubic particle with the sizes determined by the Scherrer formula. The average size of each particle was calculated as the arithmetical mean value in all directions $h k l$, since the sizes of one particle depend strongly on the $h k l$ direction. The calculated values are listed in the Table 1 . In other words, the results obtained from simulated pattern can be used as the Scherrer constant [25].

The ratio $\left(D_{a v}^{S c h}-D_{a v}^{D e b}\right) / D_{a v}^{D e b}$ characterizes the deviation between the average particle size determined by the Scherrer formula and the average size of model particle. Fig. 2 shows the error of the Scherrer formula versus the average size of model particle both with and without correction for the form-factor $K_{h k l}$. In both cases, the divergence of sizes determined by the Scherrer formula from the given size was $76 \%$ for a cubic particle consisting of 8 atoms, with edge length equal to one translation As the particle size increases, the deviation exhibited a nonlinear behavior tending to smaller values. When the particle size was more than 10 unit cell translations $(\sim 4 \mathrm{~nm})$, the error in the calculation by the original formula (1) was up to $2 \%$. If the form-factor $K_{h k l}$ is taken into account, the error is negative, which leads to a considerable particle size underestimation (over $10 \%)$.

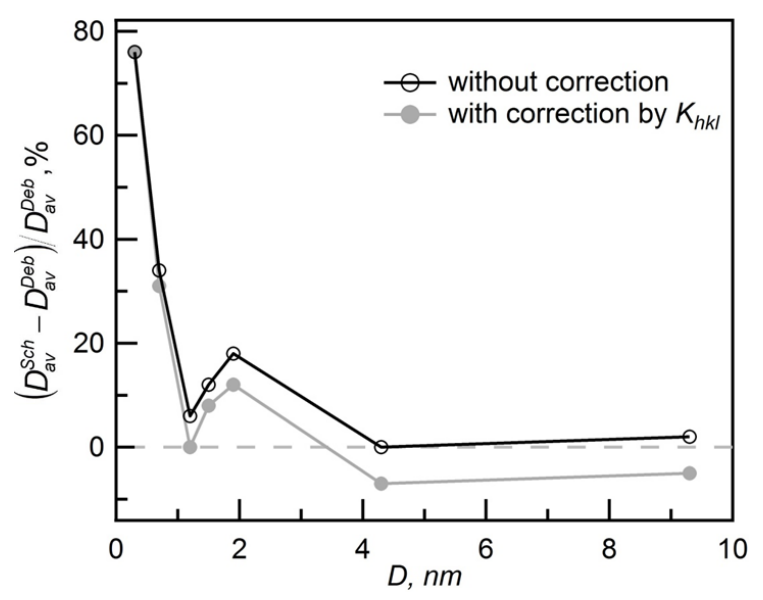

FIG. 2. The deviation of the Scherrer formula versus the size of model cubic nanoparticles determined by formula (1) and with correction for the form-factor $K_{h k l}$ by formula (2)

The Scherrer formula implies scattering on a centrosymmetric particle (sphere, for example). The size values for different $h k l$-directions vary nearby the average particle size $D_{a v}^{S c h}$ (Table 1, column 3). As particle increases (Table 1, column 1), the sizes determined at any $h k l$-directions approach the average particle size $D_{a v}^{S c h}$. On the other hand, the average size of the model particle $D_{a v}^{D e b}$ also demonstrates tendency to the size of centrosymmetric particle with increasing of number of atoms in the particle. Thus, the particle of 8 atoms is described by one size at one $h \mathrm{kl}$-direction, and the particle of 256 atoms is described by 5 sizes at $5 \mathrm{hkl}$-directions. The more items (sizes at different $h k l$ ) in the sum the closer average particle size to a value characteristic of a centrosymmetric particle. According to this, the average particle sizes $D_{a v}^{S c h}$ and $D_{a v}^{D e b}$ approach each other as the particle sizes increase, and they become an equal at value $4.27 \mathrm{~nm}$.

This implies that form-factor $K_{h k l}$ can be ignored for estimation of particle size. The main role of the form factor $K_{h k l}$ is to take into account the deviation of the particle size from centrosymmetry. The correction $K_{h k l}$ strongly underestimates the size of the cubic particle body diagonal (Table $1, h k l=111$ ), so the average particle size drops sharply (Table 1, column 4). In other $h k l$ directions, the sizes obtained with the $K_{h k l}$ correction behave ambiguously with respect to the model particle size at the same $h k l$ directions. The influence of the form factor is supposed to differ for particle size larger than $10 \mathrm{~nm}$. Nevertheless, using form factor for the refinement procedure seems to be useless for the size range below $10 \mathrm{~nm}$.

Despite the significant relative deviation of the average particle size, the absolute value of error does not exceed $0.3 \mathrm{~nm}$. As a rule, this accuracy is sufficient for powder diffraction investigations. More accurate measurement requires detailed high-resolution transmission electron microscopy. In other words, the Scherrers formula can be used even for estimation of sizes of small particle. Moreover, due to the proximity of particle sizes at different 
TABLE 1 . The particle size versus $h k l$ directions, and the average size $\bar{D}$ in all directions $h k l$

\begin{tabular}{|c|c|c|c|c|}
\hline 1 & 2 & 3 & 4 & 5 \\
\hline $\begin{array}{l}\text { Number } \\
\text { of atoms }\end{array}$ & $h k l$ & $\begin{array}{c}\text { Particle size } D^{S c h} \\
\text { by formula (1) }\end{array}$ & $\begin{array}{l}\text { Particle size } D_{h k l}^{S c h} \\
\text { with correction ( } 2 \text { ) }\end{array}$ & $\begin{array}{l}\text { Model particle size } \\
\qquad D^{D e b}=a \cdot K_{a}\end{array}$ \\
\hline 8 & 100 & 0.53 & 0.51 & 0.30 \\
\hline $\bar{D}_{8}$ & & 0.53 & 0.51 & 0.30 \\
\hline \multirow[t]{2}{*}{27} & 100 & 1.02 & 0.99 & 0.60 \\
\hline & 110 & 0.91 & 0.85 & 0.85 \\
\hline $\bar{D}_{27}$ & & 0.97 & 0.92 & 0.72 \\
\hline \multirow[t]{3}{*}{64} & 100 & 1.30 & 1.26 & 0.90 \\
\hline & 110 & 1.60 & 1.49 & 1.27 \\
\hline & 111 & 1.05 & 0.95 & 1.56 \\
\hline $\bar{D}_{64}$ & & 1.32 & 1.23 & 1.24 \\
\hline \multirow[t]{4}{*}{125} & 100 & 1.71 & 1.65 & 1.20 \\
\hline & 110 & 1.82 & 1.70 & 1.70 \\
\hline & 111 & 1.40 & 1.26 & 2.08 \\
\hline & 210 & 1.99 & 1.63 & 1.20 \\
\hline $\bar{D}_{125}$ & & 1.73 & 1.56 & 1.54 \\
\hline \multirow[t]{5}{*}{256} & 100 & 2.05 & 1.99 & 1.50 \\
\hline & 110 & 2.35 & 2.19 & 2.12 \\
\hline & 111 & 1.89 & 1.69 & 2.60 \\
\hline & 200 & 2.31 & 1.99 & 1.50 \\
\hline & 210 & 2.47 & 2.02 & 1.68 \\
\hline $\bar{D}_{256}$ & & 2.21 & 1.97 & 1.88 \\
\hline \multirow[t]{6}{*}{1728} & 100 & 4.18 & 4.04 & 3.30 \\
\hline & 110 & 4.43 & 4.13 & 4.67 \\
\hline & 111 & 4.34 & 3.89 & 5.72 \\
\hline & 200 & 4.13 & 3.54 & 3.30 \\
\hline & 210 & 4.51 & 3.69 & 3.69 \\
\hline & 211 & 4.02 & 3.12 & 4.95 \\
\hline $\bar{D}_{1728}$ & & 4.27 & 3.74 & 4.27 \\
\hline \multirow[t]{6}{*}{17576} & 100 & 9.18 & 8.88 & 7.20 \\
\hline & 110 & 9.75 & 9.09 & 10.18 \\
\hline & 111 & 9.34 & 8.36 & 12.47 \\
\hline & 200 & 9.30 & 7.98 & 7.20 \\
\hline & 210 & 9.18 & 7.52 & 8.05 \\
\hline & 211 & 10.12 & 7.87 & 10.80 \\
\hline $\bar{D}_{17576}$ & & 9.48 & 8.28 & 9.32 \\
\hline
\end{tabular}


$h k l$-directions to the average value, it is not necessary to use all peaks of diffraction pattern. The calculations show (Table 1, column 3) that applying the Scherrer formula to a single reflex of the diffraction pattern is enough to estimate the average particle size.

This is very important in cases of multiphase samples, where most reflections of different phases overlap in the experimental diffraction pattern. The possibility to use the only reflex for estimation the size of a particle with insignificant error can be great help for majority of material science researchers. Hence, in order to decrease the error of size estimation and to account the asymmetric shape of particle, the simulation of diffraction pattern by the Debye formula and high resolution electron microscopy investigation are required.

This analysis allows one to conclude that at the range below $10 \mathrm{~nm}$, the Scherrer formula without $K_{h k l^{-}}$ correction gives more exact values. If the sizes of crystal nanoparticle are less than $4 \mathrm{~nm}$, the relative error of the Scherrer formula increases appreciably. Nevertheless, the absolute error of the Scherrer formula does not exceed $0.3 \mathrm{~nm}$ for estimation of the average size of center-symmetrical particle.

\section{Acknowledgements}

The author thanks E. V. Shalaeva for useful discussion and A. L. Syuzyumova for English translation of the article. This work was supported by the Russian Science Foundation (project No. 17-79-20165) and performed at the Institute of Solid State Chemistry UrB RAS.

\section{References}

[1] Ingham B. X-ray scattering characterisation of nanoparticles. Crystallography Reviews, 2015, 21 (4), P. $229-303$.

[2] Muniz F.T.L., Miranda M.A.R., dos Santos C.M., Sasaki J.M. The Scherrer equation and the dynamical theory of X-ray diffraction. Acta Crystallographica A: Found. \& Adv, 2016, 72 (3), P. 385-390.

[3] Miranda M.A.R., Sasaki J.M. The limit of application of the Scherrer equation. Acta Crystallographica A: Found. \& Adv., 2018, 74 (1), P. 54-65.

[4] Uvarov V., Popov I. Metrological characterization of X-ray diffraction methods at different acquisition geometries for determination of crystallite size in nano-scale materials. Materials Characterization, 2013, 85, P. 111-123.

[5] Uvarov V., Popov I. Metrological characterization of X-ray diffraction methods for determination of crystallite size in nano-scale materials. Mater. Charact., 2007, 27, P. 883-891.

[6] Scardi P., Billinge S.J.L., Neder R., Cervellino A. Celebrating 100 years of the Debye scattering equation. Acta Crystallographica A: Found. \& Adv., 2016, 72 (6), P. 589-590.

[7] Hall B.D., Zanchet D., Ugarte D. Estimating nanoparticle size from diffraction measurements. J. Appl. Cryst., 2000,33 , P. $1335-1341$.

[8] Rempel A.A., Vorokh A.S., Neder R., Magerl A. Disordered structure of cadmium sulphide nanoparticles. J. Surf. Investig. X-ray, Synchr. and Neutron Tech., 2011, 5 (6), P. 1028-1031.

[9] Vorokh A.S., Rempel A.A. Direct-space visualization of the short and average long-range orders in the noncrystalline sctructure of a single cadmium sulfide nanoparticle. JETP Letters, 2010, 91 (2), P. 100-104.

[10] Perez-Demydenko C., Scardi P. Diffraction peak profiles of surface relaxed spherical nanocrystals. Philosophical Magazine, 2017, 97 (26),P. 2317-2346.

[11] Sestu M., Navarra G., et al. Whole-nanoparticle atomistic modeling of the schwertmannite structure from total scattering data. J. Appl. Cryst., 2017, 50, P. 1617-1626.

[12] Tsybulya S.V., Yatsenko D.A. X-ray diffraction analysis of ultradisperse systems: The Debye formula. J. Struct. Chem., 2012, 53, S150-S165.

[13] Yatsenko D., Tsybulya S. DIANNA (diffraction analysis of nanopowders) - a software for structural analysis of nanosized powders. Zeitschrift Fur Kristallographie - Crystalline Materials, 2018, 233 (1), P. 61-66.

[14] Cervellino A., Frison R., Bertolotti F., Guagliardi A. DEBUSSY 2.0: the new release of a Debye user system for nanocrystalline and/or disordered materials. J. Appl. Cryst., 2015, 48 (6), P. 2026-2032.

[15] Nikulina O., Yatsenko D., et al. Debye function analysis of nanocrystalline gallium oxide gamma-Ga $\mathrm{O}_{3}$. Zeitschrift Fur Kristallographie Crystalline Materials, 2016, 231 (5), P. 261-266.

[16] Scherrer P. Bestimmung der Größe und der inneren Struktur von Kolloidteilchen mittels Röntgenstrahlen. Nachrichten Gesellschaft Wissenschaft Gottingen, 1918, 2, P. 98-100.

[17] Seljakow N. Eine röntgenographische Methode zur Messung der absoluten Dimensionen einzelner Kristalle in Körpern von fein kristallinischem Bau. Zeitschrift fuer Physik, 1925, 516, P. 439-444.

[18] Langford J.I., Wilson A.J.C. Scherrer after Sixty Years: A Survey and Some New Results in the Determination of Crystallite Size. J. Appl. Cryst., 1978, 11, P. 102-113.

[19] James R.W. Optical principles of the Diffraction of X Rays. G. Bell \& Sons, 1948, $624 \mathrm{p}$.

[20] Debye P. Zerstreuung von Röntgenstrahlen. Annalen der Physik B, 1915, 46, P. 809-823.

[21] International Tables for X Ray crystallography IV, Birmingham, England, 1974, 366 p.

[22] Mitra G.B. Moments and Cumulants of Diffraction Profiles Broadened by Stacking Faults. Journal of Crystallization Process and Technology, 2013, 3, P. 103-107.

[23] Ectors D., Goetz-Neunhoeffer F., Neubauer J. Routine (an)isotropic crystallite size analysis in the double-Voigt approximation done right? Powder Diffraction, 2017, 32, S27-S34.

[24] Ida T. New measures of sharpness for symmetric powder diffraction peak profiles. J. Appl. Cryst., 2008,41, P. $393-401$.

[25] Li Z. Characterization of Different Shaped Nanocrystallites using X-ray Diffraction Line Profiles. Particle \& Particle Systems Characterization, 2011, 28 (1-2), P. 19-24. 